\title{
Attractors with irrational rotation number
}

\author{
Luis Hernández-Corbato, Rafael Ortega, Francisco R. Ruiz del Portal *
}

September 2, 2010

\begin{abstract}
Let $h: \mathbb{R}^{2} \rightarrow \mathbb{R}^{2}$ be a dissipative and orientation preserving homeomorphism having an asymptotically stable fixed point $p$. Let $U$ be the region of attraction and assume that it is proper and unbounded. Using Carathéodory's prime ends theory one can associate a rotation number, $\rho$, to $h_{\mid U}$. We prove that any map in the above conditions and with $\rho \notin \mathbb{Q}$ induces a Denjoy homeomorphism in the circle of prime ends. We also present some explicit examples of maps in this class and we show that, if the infinity point is accessible by an arc in $U, \rho \notin \mathbb{Q}$ whenever $\operatorname{Per}(h)=\operatorname{Fix}(h)=\{p\}$.
\end{abstract}

\section{Introduction}

In this paper we continue the work initiated in [14] on the connections between Rotation Numbers and Stability Theory. As in the previous paper we assume that $h$ is a dissipative homeomorphism of the plane having an assymptotically stable fixed point, say $x_{*}=h\left(x_{*}\right)$. Moreover it will be assumed that the region of attraction

$$
U=\left\{x \in \mathbb{R}^{2}: h^{n}(x) \rightarrow x_{*} \text { as } n \rightarrow+\infty\right\}
$$

is unbounded.

We recall that $h$ is dissipative if there exist a compact set $B \subset \mathbb{R}^{2}$ such that $h(B) \subset \operatorname{int}(B)$ and all orbits are attracted by $B$. This attraction is uniform in compact sets. The notion of dissipativity appeared first in the theory of Nonlinear Oscillations but now it is used in many contexts. See

${ }^{*}$ The first and third authors have been supported by MEC, MTM2009-07030, the second by MEC, MTM 2008-02502.

2000 Mathematics Subject Classification: 37C25, 37B30, 54H25.

Keywords and phrases. Carathéodory's prime ends, attractors, dissipative homeomorphisms, rotation number, Denjoy homeomorphism. 
[10] and [8] for more information. The assumption on the unboundedness of $U$ can be checked in many concrete cases. In particular it is automatic when $h$ is area-contracting.

The region of attraction is an open and simply connected subset of $\mathbb{R}^{2}$ that is invariant under $h$. When the point $x_{*}$ is not a global attractor, $U$ is a proper subset of the plane and the theory of Prime Ends due to Carathéodory can be applied. Even if the boundary of $U$ is very complicated, the space of prime ends $\mathbb{P}=\mathbb{P}(U)$ is always homeomorphic to a circumference so that $U^{\star}=U \cup \mathbb{P}$ becomes a closed disk. The effect of $h$ on prime ends induces a map $h^{\star}: \mathbb{P} \rightarrow \mathbb{P}$. This new map is always a homeomorphism and it is orientation-preserving as soon as $h$ has this property in $\mathbb{R}^{2}$. In such a case $h^{\star}$ has a rotation number that will be denoted by $\rho=\rho(h, U)$. Typically rotation numbers must be interpreted as angles and we will assume that $\rho$ belongs to $\mathbb{T}=\mathbb{R} / \mathbb{Z}$, or sometimes $\rho \in[0,1)$. The idea of assigning a rotation number to a planar invariant region is classical in Dynamical Systems and goes back to Birkhoff. We refer to the papers $[4,7,2]$ for more information. In the previous paper [14] the case $\rho=0$ (or more generally $\rho \in \mathbb{Q}$ ) was treated, now we plan to study the case $\rho \in \mathbb{R} \backslash \mathbb{Q}$.

The first question is the existence of homeomorphisms in the above conditions having an irrational rotation number. We will construct explicit examples using the well-known theory of Denjoy, which produces homeomorphisms of $\mathbb{S}^{1}$ that are not conjugate to a rotation but have irrational rotation number. It is well known that these Denjoy homeomorphisms cannot be very smooth and so our map $h$ will not be smooth either. In principle it could be thought that this association between irrational rotation numbers and Denjoy maps is not essential and perhaps due to our specific construction. Indeed this is not the case since we will prove that irrational rotations cannot appear on prime ends. For any $h$ in the above conditions with irrational $\rho$, the induced map $h^{\star}$ is a Denjoy homeomorphism. The appearance of Denjoy dynamics in spaces of prime ends has been found by Barge in his study of Hénon map. See [3] and also [13] for other related results.

Besides the connection with Denjoy theory, the examples constructed in this paper have another feature: they possess a local attractor and no periodic points. This is of some relevance for the general question on how to pass from local to global attraction. Given a homeomorphism in arbitrary dimension $h: \mathbb{R}^{d} \rightarrow \mathbb{R}^{d}$ with an assymptotically stable fixed point $x_{*}=$ $h\left(x_{*}\right)$, the global attraction of $x_{*}$ is characterized by the properties

$h$ is dissipative and $\operatorname{Rec}(h)=\left\{x_{*}\right\}$,

where $\operatorname{Rec}(h)$ is the set of recurrent points of $h$. In low dimension this result can be sharpened. For $d=1$ it is sufficient to exclude 2-cycles and the global attraction is equivalent to

$$
h \text { is dissipative and } \operatorname{Fix}\left(h^{2}\right)=\left\{x_{*}\right\} .
$$

It was proved in [14] that this is also a characterization of global attraction for $d=2$ if $h$ is orientation-reversing. In this setting Bonino proved in 
[6] that the existence of a $k$-cycle with $k \geq 2$ implies the existence of a 2cycle. Hence Fix $\left(h^{2}\right)=\left\{x_{*}\right\}$ is indeed equivalent to $\operatorname{Per}(h)=\left\{x_{*}\right\}$, where $\operatorname{Per}(h)$ is the set of periodic points. This is in contrast with the orientationpreserving case, we construct examples with $U \neq \mathbb{R}^{2}$ and satisfying

$$
h \text { is dissipative and } \operatorname{Per}(h)=\left\{x_{*}\right\} \text {. }
$$

It is interesting to notice that in the examples we construct the set $\operatorname{Rec}(h) \backslash\left\{x_{*}\right\}$ is a Cantor set with almost automorphic dynamics.

Throughout the paper we employ the theory of prime ends as presented by Pommerenke in [15]. The notations are those introduced in [14]. The class of maps under study will be denoted by $\mathcal{H}_{0}$. These maps are orientationpreserving homeomorphisms $h: \mathbb{R}^{2} \rightarrow \mathbb{R}^{2}$ satisfying

(i) $h$ is dissipative.

(ii) The origin $x_{*}=0$ is an assymptotically stable fixed point.

(iii) The region of attraction $U$ is a proper and unbounded subset of $\mathbb{R}^{2}$.

The rest of the paper is organized in five Sections. The next Section is a short description of the classification of Denjoy maps obtained by Markley in [11]. This will be useful for the rest of the paper. Sections 3 and 4 are dedicated to the construction of maps $h \in \mathcal{H}_{0}$ having irrational rotation number. In particular it will be shown that every Denjoy map can be realized in the space of prime ends by some $h^{\star}$. In Section 5 we prove that $h^{\star}$ cannot be an irrational rotation if $h \in \mathcal{H}_{0}$. Finally, in Section 6 we present some work under construction. In all the examples we know of maps $h \in \mathcal{H}_{0}$ with $\rho(h, U) \notin \mathbb{Q}$, the set of periodic points is simply

$$
\operatorname{Per}(h)=\{0\} .
$$

It seems plausible that this could be a characterization of irrational rotation numbers in the class $\mathcal{H}_{0}$. By now we have a proof with an additional assumption on $U$. The paper is finished with an appendix containing proofs of several lemmas on Denjoy maps.

\section{The classification of Denjoy homeomorphisms}

In this Section we review results obtained by Markley in [11]. We work in the quotient space $\mathbb{T}=\mathbb{R} / \mathbb{Z}$ with points

$$
\bar{\theta}=\theta+\mathbb{Z}, \quad \theta \in \mathbb{R} .
$$

The distance between $\bar{\theta}_{1}$ and $\bar{\theta}_{2}$ is

$$
d\left(\bar{\theta}_{1}, \bar{\theta}_{2}\right)=\operatorname{dist}_{\mathbb{R}}\left(\theta_{1}-\theta_{2}, \mathbb{Z}\right),
$$


where dist $_{\mathbb{R}}$ indicates the distance from a point to a set on the real line. Closed arcs will be indicated by greek letters $\alpha, \beta, .$. and the corresponding open arcs will be $\dot{\alpha}, \dot{\beta}, \ldots$. Given $\eta \in \mathbb{R}$ we consider the rotation $R_{\eta}: \mathbb{T} \rightarrow$ $\mathrm{T}, R_{\eta}(\bar{\theta})=\overline{\theta+\eta}$. Two sets $S_{1}$ and $S_{2}$ in $\mathrm{T}$ are congruent $\left(S_{1} \equiv S_{2}\right)$ if $R_{\eta}\left(S_{1}\right)=S_{2}$ for some $\eta \in \mathbb{R}$.

We have chosen to work on the space $\mathbb{T}$, but all figures will be sketched on the unit circle

$$
\mathbb{S}^{1}=\{z \in \mathbb{C}:|z|=1\} .
$$

Given a Cantor set $C$ in $\mathbb{T}$, we split it into the accessible and inaccessible sets,

$$
C=A \cup I, \quad A \cap I=\emptyset .
$$

The complement of $C$ can be described as

$$
\mathbb{T} \backslash C=\bigcup_{k=0}^{\infty} \dot{\alpha}_{k}
$$

where $\left(\alpha_{k}\right)_{k \geq 0}$ is a family of pairwise disjoint closed arcs. The accessible set $A$ is composed by the end points of all $\alpha_{k}$. A Cantor function associated to $C$ is a continuous function $\mathcal{P}: \mathbb{T} \rightarrow \mathbb{T}$ satisfying

$$
\mathcal{P}\left(\bar{\theta}_{1}\right)=\mathcal{P}\left(\bar{\theta}_{2}\right) \Leftrightarrow \bar{\theta}_{1}=\bar{\theta}_{2} \text { or } \bar{\theta}_{1}, \bar{\theta}_{2} \in \alpha_{k} \text { for some } k \geq 0 .
$$

To visualize this type of maps we collapse every arc $\alpha_{k}$ into a point in such a way that the cyclic order is preserved.
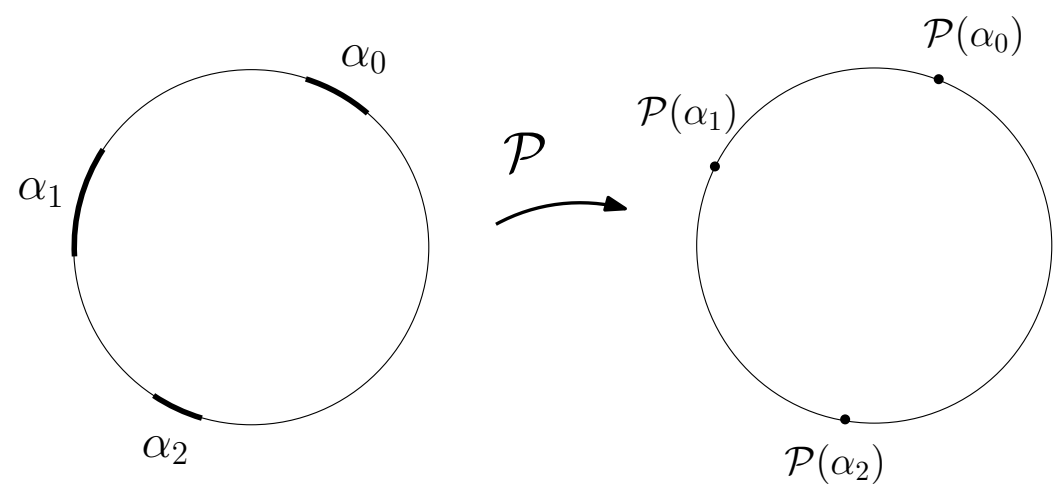

Figure 1: Example of Cantor function.

It is not hard to prove that $\mathcal{P}$ is onto and $\mathcal{P}(A)$ is a countable and dense subset of $\mathbb{T}$.

A Denjoy map is an orientation-preserving homeomorphism $\phi: \mathbb{T} \rightarrow \mathbb{T}$ having an irrational rotation number and not conjugate to a rotation. From now on the rotation number of $\phi$ will be $\bar{\omega}$, with $\omega \in \mathbb{R} \backslash \mathbb{Q}$. Since $\phi$ is not 
conjugate to $R_{\omega}$ we know that there exists a Cantor set $C=C_{\phi}$ contained in $\mathbb{T}$ which is minimal for $\phi$ and attracts all orbits, in the future and in the past. Moreover there exists a Cantor function $\mathcal{P}$ associated to $C_{\phi}$ and such that $\mathcal{P} \circ \phi=R_{\omega} \circ \mathcal{P}$. A key fact for the classification of Denjoy maps is the uniqueness of this $\mathcal{P}$ up to rotations.

Lemma 1. Assume that $\mathcal{P}_{1}$ and $\mathcal{P}_{2}$ are Cantor functions associated to $C_{\phi}$ and such that

$$
\mathcal{P}_{i} \circ \phi=R_{\omega} \circ \mathcal{P}_{i}, i=1,2 .
$$

Then there exists $\eta \in \mathbb{R}$ such that $\mathcal{P}_{2}=R_{\eta} \circ \mathcal{P}_{1}$.

Proof. After composing with a rotation we can assume that $\mathcal{P}_{1}\left(\alpha_{0}\right)=\mathcal{P}_{2}\left(\alpha_{0}\right)$. Let us prove that $\mathcal{P}_{1}$ and $\mathcal{P}_{2}$ coincide in this case.

The invariance of $C$ under $\phi$ implies that the iterated $\operatorname{arcs} \phi^{n}\left(\alpha_{0}\right)$ must become a sub-family of $\left(\alpha_{k}\right)_{k \geq 0}$. We employ the notation

$$
\alpha_{\sigma(n)}=\phi^{n}\left(\alpha_{0}\right), \quad n \in \mathbb{Z}
$$

and notice that

$$
\mathcal{P}_{i}(\bar{\theta})=\mathcal{P}_{i}\left(\phi^{n}\left(\alpha_{0}\right)\right)=R_{\omega}^{n}\left(\mathcal{P}_{i}\left(\alpha_{0}\right)\right), \quad i=1,2
$$

whenever $\bar{\theta} \in \alpha_{\sigma(n)}$. Thus $\mathcal{P}_{1}=\mathcal{P}_{2}$ on the set $\bigcup_{n \in \mathbb{Z}} \alpha_{\sigma(n)}$. Since this set is invariant under $\phi$, its closure must contain $C$. Therefore $\mathcal{P}_{1}$ and $\mathcal{P}_{2}$ coincide on $C$ and the conclusion follows from the definition of Cantor function.

Given $\phi, C=A \cup I$ and $\mathcal{P}$ as before, we notice that $\mathcal{P}(A)$ is a nonempty, countable set satisfying $R_{\omega}(\mathcal{P}(A))=\mathcal{P}(A)$. This set is not uniquely determined by $\phi$, but the previous result implies that the class of congruence of $\mathcal{P}(A)$ is unique. In general, given two Denjoy maps $\phi_{1}$ and $\phi_{2}$ which are topologically conjugate, Lemma 1 implies that $\mathcal{P}_{1}\left(A_{1}\right) \equiv \mathcal{P}_{2}\left(A_{2}\right)$. In [11] it is proved that the converse is also valid and so the rotation number $\bar{\omega}$ and the class of congruence of $\mathcal{P}(A)$ are sufficient to classify Denjoy maps. Actually the paper [11] contains a complete topological description of Denjoy maps: given $\omega \in \mathbb{R} \backslash \mathbb{Q}$ and $M \subset \mathbb{T}$ with $M \neq \emptyset, M$ countable and $R_{\omega}(M)=M$, there exists a Denjoy map $\phi$ with rotation number $\bar{\omega}$ and such that $\mathcal{P}(A)=$ $M$.

It must be noticed that Markley originally worked with $\mathcal{P}(I)$ instead of $\mathcal{P}(A)$. These are obviously equivalent invariants but we find more intuitive to think in terms of the countable set $\mathcal{P}(A)$. The above discussions will be illustrated with the intuitive construction of one of the simplest Denjoy maps. The standard construction appearing in textbooks such as [1] corresponds to a set $\mathcal{P}(A)$ composed by a unique orbit of $R_{\omega}$, say

$$
\mathcal{P}(A)=\{\overline{\varphi+n \omega}: n \in \mathbb{Z}\}
$$


with $\varphi \in[0,1)$. Let us discuss the case of two orbits

$$
\mathcal{P}(A)=\{\overline{\varphi+n \omega}: n \in \mathbb{Z}\} \cup\{\overline{\psi+n \omega}: n \in \mathbb{Z}\}
$$

with $\varphi, \psi \in[0,1), \varphi<\psi$. We start with a Cantor set $C$ in $\mathbb{T}$ and split the family of $\operatorname{arcs}\left(\alpha_{k}\right)_{k \geq 0}$ in two sub-families $\left(\beta_{n, \varphi}\right)_{n \in \mathbb{Z}}$ and $\left(\beta_{n, \psi}\right)_{n \in \mathbb{Z}}$ satisfying

$$
\begin{array}{cc}
\phi\left(\beta_{n, \varphi}\right)=\beta_{n+1, \psi}, & \phi\left(\beta_{n, \psi}\right)=\beta_{n+1, \psi}, \\
\mathcal{P}\left(\beta_{n, \varphi}\right)=\overline{\varphi+n \omega}, & \mathcal{P}\left(\beta_{n, \psi}\right)=\overline{\psi+n \omega},
\end{array}
$$

and such that the cyclic order is preserved by $\phi$ and $\mathcal{P}$,


Figure 2: Cantor function constructed.

To finish this Section we generalize the previous procedure and describe how to label the family of arcs $\left(\alpha_{k}\right)$ according to the dynamics. Assume now that $\phi$ is an arbitrary Denjoy map and $C=A \cup I$ and $\mathcal{P}$ have the obvious meanings. The set $\mathcal{P}(A)$ being countable and invariant under $R_{\omega}$, it can be described as a disjoint union of orbits of $R_{\omega}$. Let us pick exactly one point in each of these orbits to obtain a family of points

$$
\left\{\overline{\varphi_{\lambda}}: \lambda \in \Lambda\right\}
$$

where $\overline{\varphi_{\lambda}} \in \mathcal{P}(A), \Lambda$ is a non-empty countable set and

$$
\mathcal{P}(A)=\left\{\overline{\varphi_{\lambda}+n \omega}: \lambda \in \Lambda, n \in \mathbb{Z}\right\} .
$$

Moreover $(\lambda, n) \mapsto \overline{\varphi_{\lambda}+n \omega}$ is one-to-one. Notice that in the previous example $\Lambda$ had two elements. Define $\beta_{n, \lambda}=\mathcal{P}^{-1}\left(\overline{\varphi_{\lambda}+n \omega}\right)$. Then the family of $\operatorname{arcs}\left(\beta_{n, \lambda}\right)_{n \in \mathbb{Z}, \lambda \in \Lambda}$ coincides with $\left(\alpha_{k}\right)_{k \geq 0}$ and the identity

$$
\phi\left(\beta_{n, \lambda}\right)=\beta_{n+1, \lambda}
$$

holds. 


\section{$3 \quad$ Denjoy maps and prime ends}

Maps in the class $\mathcal{H}_{0}$ with any rational rotation number $\rho(h, U)$ are easy to construct. As an example consider $h=R \circ \phi_{1}$, where $R$ is the rotation of 90 degrees and $\phi_{1}$ is the time 1 map associated to the flow $\left\{\phi_{t}\right\}$ indicated in the figure below.

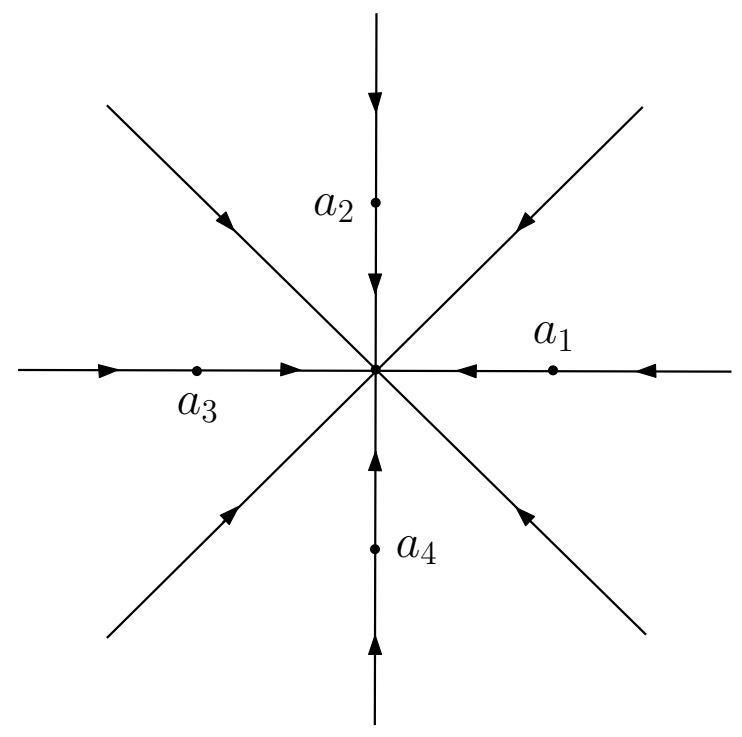

The region of attraction is the whole plane excepting the four points $a_{1}, . ., a_{4}$ and the orbits attracted by them. The space of prime ends is described in the figure below

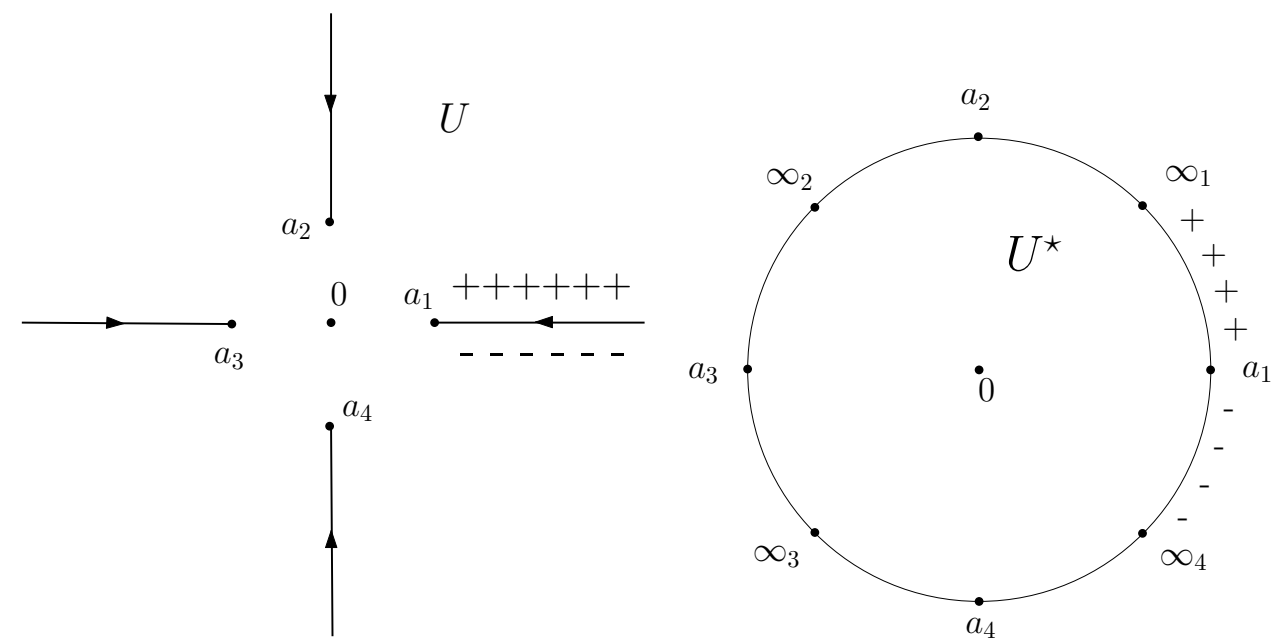

The induced map $h^{\star}$ has two 4-cycles, the attractor $\left\{a_{1}, a_{2}, a_{3}, a_{4}\right\}$ and 
the repeller $\left\{\infty_{1}, \infty_{2}, \infty_{3}, \infty_{4}\right\}$. The rotation number can be $\frac{1}{4}$ or $\frac{3}{4}$ depending on the sense of rotation of $R$.

The purpose of this Section is to present some maps in $\mathcal{H}_{0}$ with $\rho \in \mathbb{R} \backslash \mathbb{Q}$. In particular we will prove the following result.

Proposition 2. Given $\omega \in(0,1) \backslash \mathbb{Q}$ and a Denjoy map $\phi$, there exists $h \in \mathcal{H}_{0}$ with rotation number $\rho(h, U)=\bar{\omega}$ and such that $h^{\star}$ is topologically conjugate to $\phi$.

The proof of this result will consist in an explicit construction of $h$ based on $\phi$ and the function $\Omega$ given by the following lemma.

Lemma 3. Given a Denjoy map $\phi$ with minimal Cantor set $C$, there exists a continuous function $\Omega: \mathbb{T} \rightarrow \mathbb{R}$ satisfying

(i) $0 \leq \Omega(\bar{\theta})<\frac{3}{4}$ for each $\bar{\theta} \in \mathbb{T}$

(ii) $\Omega^{-1}(0)=C$

(iii) The series $\sum_{n \geq 0} \Omega\left(\phi^{n}(\bar{\theta})\right)$ and $\sum_{n \leq 0} \Omega\left(\phi^{n}(\bar{\theta})\right)$ are divergent if $\bar{\theta} \in$ $\mathrm{T} \backslash C$.

The proof of this auxiliary result is postponed to the Appendix. We define $h$ in polar coordinates $\theta$ and $\rho$. The same notation will be employed for a function defined on $\mathbb{T}$ and its lift defined on $\mathbb{R}$. Define

$$
h: \theta_{1}=\phi(\theta), \quad \rho_{1}=R(\theta, \rho)
$$

where $R: \mathbb{T} \times[0,+\infty) \rightarrow[0,+\infty)$ is the function such that $R(\theta, \cdot)$ is piecewise linear, with corner points at $\rho=\frac{1}{2}$ and $\rho=1$ and satisfying

$$
\begin{aligned}
R(\theta, 0)=0, \quad R\left(\theta, \frac{1}{2}\right) & =\frac{1}{4}, \quad R(\theta, 1)=1-\Omega(\theta) \\
\frac{\partial R}{\partial \rho}(\theta, \rho) & =\frac{1}{2} \text { if } \rho>1 .
\end{aligned}
$$

For later computations it is convenient to present $R$ more explicitly,

$$
R(\theta, \rho)= \begin{cases}\frac{1}{2} \rho & \text { if } \rho \leq \frac{1}{2} \\ \left(\frac{3}{4}-\Omega(\theta)\right)(2 \rho-1)+\frac{1}{4} & \text { if } \frac{1}{2}<\rho \leq 1 \\ \frac{1}{2} \rho+\frac{1}{2}-\Omega(\theta) & \text { if } \rho>1\end{cases}
$$

It is easy to prove that $h$ is an orientation-preserving homeomorphism with $h(0)=0$. To prove the dissipativity it is sufficient to invoke the inequality

$$
\rho_{1} \leq \frac{1}{2} \rho+\frac{1}{2} \quad \text { if } \rho \geq 1
$$


It implies that every disk $\rho \leq r$ with radius $r>1$ is mapped into its interior. Moreover every orbit in $\mathbb{R}^{2}$ is attracted (uniformly on compact sets) by any of these disks.

Similarly the inequality

$$
\rho_{1}<\rho \text { if } 0<\rho<1
$$

can be employed to prove that the origin is asymptotically stable and the open disk $\rho<1$ is contained in $U$. Indeed it is possible to give an exact description of the region of attraction. We claim that

$$
U=\mathbb{R}^{2} \backslash\{(\bar{\theta}, \rho): \bar{\theta} \in C, \rho \geq 1\} .
$$

Assume first that $\left(\bar{\theta}_{0}, \rho_{0}\right)$ is a point with $\bar{\theta}_{0} \in C$ and $\rho_{0} \geq 1$ and let $\left\{\left(\bar{\theta}_{n}, \rho_{n}\right)\right\}$ be the corresponding orbit. The invariance of $C$ under $\phi$ implies that $\Omega\left(\theta_{n}\right)=0$ for each $n$ and so

$$
\rho_{n+1}=\frac{1}{2} \rho_{n}+\frac{1}{2}, \quad n \in \mathbb{Z} .
$$

This implies that $\rho_{n}$ decreases to 1 as $n \rightarrow+\infty$ and so $\left(\bar{\theta}_{0}, \rho_{0}\right)$ is not in $U$. To prove the reversed inclusion in (1) we take an orbit $\left\{\left(\bar{\theta}_{n}, \rho_{n}\right)\right\}$ with $\bar{\theta}_{0} \notin C$ and prove that some iterate must enter into the disk $\rho<1$. This is sufficient to guarantee that $\left(\bar{\theta}_{0}, \rho_{0}\right)$ is in $U$. Assuming by contradiction that $\rho_{n} \geq 1$ for each $n \geq 0$ one is lead to

$$
\rho_{n+1}=\frac{1}{2} \rho_{n}+\frac{1}{2}-\Omega\left(\theta_{n}\right) \leq \rho_{n}-\Omega\left(\theta_{n}\right), \quad n \geq 0 .
$$

This implies

$$
\rho_{n} \leq \rho_{0}-\sum_{k=0}^{n-1} \Omega\left(\phi^{k}\left(\theta_{0}\right)\right)
$$

and so $\rho_{n}$ should become negative for large $n$. This argument completes the proof of (1).

Now it is clear that $h \in \mathcal{H}_{0}$ since $U$ is an unbounded subset of $\mathbb{R}^{2}$ with $U \neq \mathbb{R}^{2}$. Our next task is to describe the space of prime ends $\mathbb{P}=\mathbb{P}(U)$. The boundary of $U$ in the Riemann sphere $\mathbb{S}^{2}=\mathbb{R}^{2} \cup\{\infty\}$ is composed by the rays $\{(\bar{\theta}, \rho): \rho \geq 1\}$ with $\bar{\theta} \in C$ together with the point of infinity. The set of points in $\partial_{\mathbb{S}^{2}} U$ that are accessible from $U$ is $A_{1} \cup A_{2} \cup\{\infty\}$ with

$$
A_{1}=\{(\bar{\theta}, 1): \bar{\theta} \in C\}, A_{2}=\{(\bar{\theta}, \rho): \bar{\theta} \in A, r>1\}
$$

Every prime end is obtained via an arc tending to one of these points. The prime ends associated to $A_{1}$ are such that

$$
\Pi\left(\mathfrak{p}_{1}\right)=\{(\bar{\theta}, 1)\} \text { and } I\left(\mathfrak{p}_{1}\right)=\{(\bar{\theta}, r): r \geq 1\} \cup\{\infty\},
$$




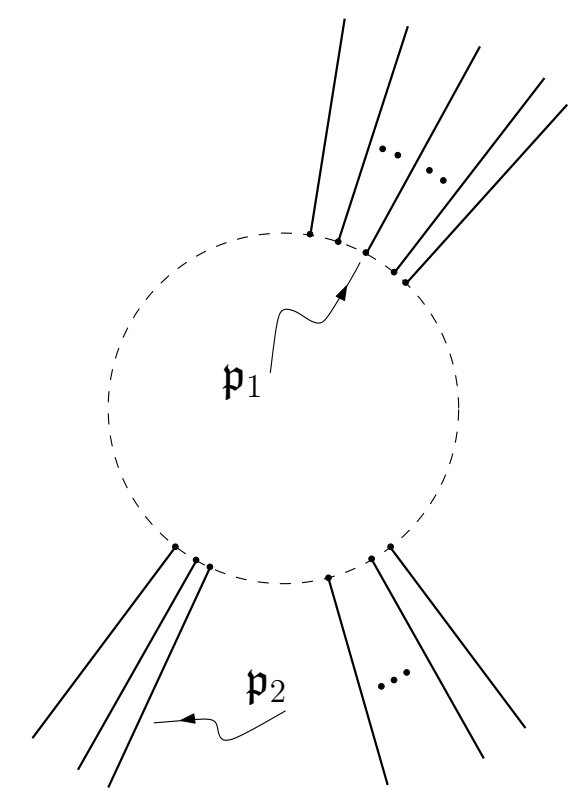

where $\Pi\left(\mathfrak{p}_{1}\right)$ is the set of principal points and $I\left(\mathfrak{p}_{1}\right)$ is the impression of $\mathfrak{p}_{1}$ (see [15] or [12] for more information on these sets). Prime ends associated to $A_{2}$ satisfy

$$
\Pi\left(\mathfrak{p}_{2}\right)=I\left(\mathfrak{p}_{2}\right)=\{(\bar{\theta}, r)\} .
$$

Finally we denote by $\infty_{n, \lambda}$ the prime end associated to the chain of cross cuts

$$
C_{k}=\left\{(\bar{\theta}, k): \bar{\theta} \in \beta_{n, \lambda}\right\}, \quad k=1,2, . .
$$

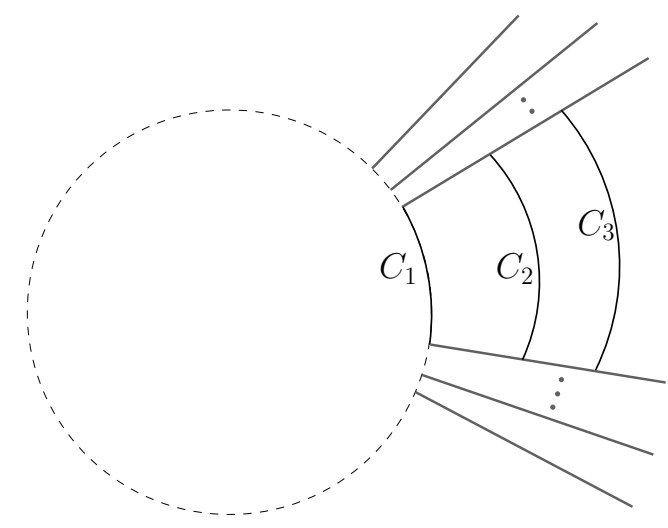

Notice that $\Pi\left(\infty_{n, \lambda}\right)=I\left(\infty_{n, \lambda}\right)=\{\infty\}$.

Once we understand the structure of $\mathbb{P}$ we can describe the dynamics of $h^{\star}$. Given an $\operatorname{arc} \beta_{n, \lambda}$ with end points $a=a_{n, \lambda}, b=b_{n, \lambda}$, we consider the 
$\operatorname{arc} \hat{\beta}_{n, \lambda}$ in $\mathbb{P}$ having end points at the corresponding prime ends $\hat{a}, \hat{b}$ and passing through $\infty_{n, \lambda}$. The next figure illustrates this definition.
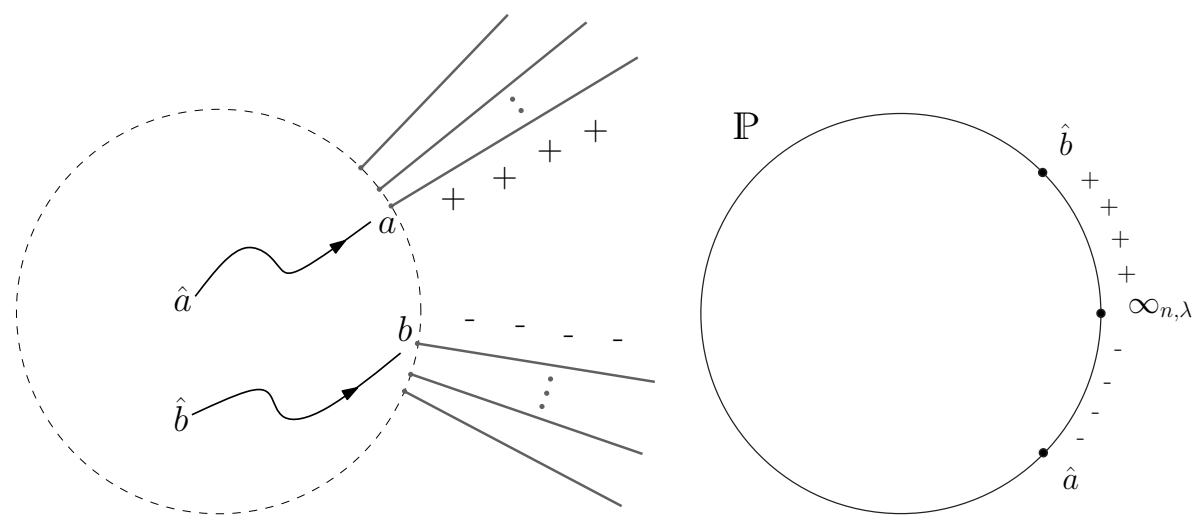

We know that $\phi\left(\beta_{n, \lambda}\right)=\beta_{n+1, \lambda}$ and this implies that $h^{\star}\left(\hat{\beta}_{n, \lambda}\right)=\hat{\beta}_{n+1, \lambda}$. Let $\mathfrak{p}_{\bar{\theta}}$ denote the prime end with principal point $(\bar{\theta}, 1)$ with $\bar{\theta} \in C$. Then

$$
\hat{C}=\left\{\mathfrak{p}_{\bar{\theta}}: \bar{\theta} \in C\right\}
$$

is a Cantor set invariant under $h^{\star}$. From here it is easy to conclude that $h^{\star}$ is a Denjoy map with rotation number $\omega$. To prove the conjugacy of $h^{\star}$ and $\phi$ it is sufficient to compute Markley's invariant for $h^{\star}$. Let $\mathcal{P}: \mathbb{T} \rightarrow \mathrm{T}$ be a Cantor function associated to $\phi$, so that $\mathcal{P} \circ \phi=R_{\omega} \circ \mathcal{P}$. Define

$$
\mathcal{P}^{\star}: \mathbb{P} \rightarrow \mathbb{T}, \quad \mathcal{P}^{\star}(\mathfrak{p})= \begin{cases}\mathcal{P}(\bar{\theta}) & \text { if } \Pi(\mathfrak{p})=\{(\bar{\theta}, r))\} \\ \mathcal{P}\left(\beta_{n, \lambda}\right) & \text { if } \mathfrak{p}=\infty_{n, \lambda}\end{cases}
$$

This is a Cantor function associated to $\hat{C}$ with $\mathcal{P}^{\star} \circ h^{\star}=R_{\omega} \circ \mathcal{P}^{\star}$. The accessible set $\hat{A}=\left\{\mathfrak{p}_{\bar{\theta}}: \bar{\theta} \in A\right\}$ is mapped by $\mathcal{P}^{\star}$ onto $\mathcal{P}^{\star}(\hat{A})=\mathcal{P}(A)$ and so the conjugacy follows. The proof of Proposition 2 is now complete.

It is interesting to notice that $h$ has no periodic orbits (excepting for the origin) and that $\infty$ is accessible from $U$. The boundary in $\mathbb{R}^{2}$ of the region of attraction has infinitely many connected components. This is consistent with Theorem 10 in [14]. There it is proved that if $\infty$ is accessible from $U$ and $\partial U$ has a finite number of components then there is a periodic orbit in $\mathbb{R}^{2} \backslash U$.

\section{An alternative construction}

We will construct a new homeomorphism $h_{\text {new }} \in \mathcal{H}_{0}$ in the conditions of Proposition 2 and such that the region of attraction $U_{\text {new }}$ has a connected boundary in $\mathbb{R}^{2}$. Moreover the point of infinity will not be accessible from $U_{\text {new }}$. 
We start again with the Denjoy map $\phi$ and the function $\Omega$ given by Lemma 3. The sets

$$
F_{+}=\bigcup_{\substack{n \geq 0 \\ \lambda \in \Lambda}} \beta_{n, \lambda} \cup C, \quad F_{-}=\bigcup_{\substack{n<0 \\ \lambda \in \Lambda}} \beta_{n, \lambda} \cup C
$$

are closed in $\mathbb{T}$. To justify this it is enough to recall that $C$ is the limit set of any orbit of $\phi$. The function $\Omega$ vanishes on $F_{+} \cap F_{-}=C$ and so the function

$$
\Omega_{\text {new }}(\bar{\theta})= \begin{cases}\Omega(\bar{\theta}) & \text { if } \bar{\theta} \in F_{-} \\ 0 & \text { if } \bar{\theta} \in F_{+}\end{cases}
$$

is continuous. Replacing $\Omega$ by $\Omega_{\text {new }}$ the construction of $h$ can be repeated and a new map

$$
h_{\text {new }}: \theta_{1}=\phi(\theta), \quad \rho_{1}=R_{\text {new }}(\theta, \rho)
$$

is obtained. As before, it can be proven that $h_{\text {new }} \in \mathcal{H}_{0}$, the only essential differences being in (iii). We claim that the region of attraction can be described as

$$
U_{\text {new }}=\{(\bar{\theta}, \rho): \rho<\sigma(\bar{\theta})\}
$$

where $\sigma: \mathbb{T} \rightarrow \mathbb{R}$ is a function satisfying

$$
\sigma \geq 1 \text { everywhere and } \sup _{\mathbb{T}} \sigma=+\infty \text {. }
$$

This implies that $U_{\text {new }}$ is a proper and unbounded subset of $\mathbb{R}^{2}$ and so (iii) holds.

We find $\sigma$ in several steps. First notice that the open disk $\Delta=\{\rho<1\}$ is contained in $U_{\text {new }}$. Next, we prove that

$$
U_{\text {new }} \cap\left\{\bar{\theta} \in F_{+}\right\} \subset \Delta .
$$

This is a consequence of the positive invariance under $h_{\text {new }}$ of the set

$$
M=\left\{(\bar{\theta}, \rho): \bar{\theta} \in F_{+}, \rho \geq 1\right\} .
$$

The inclusion $h_{\text {new }}(M) \subset M$ is proved using $\phi\left(F_{+}\right) \subset F_{+}, \Omega=0$ on $F_{+}$ and the definition of $h_{\text {new }}$.

After having checked (2) we must analyze $U_{\text {new }} \cap\left\{\bar{\theta} \in F_{-}\right\}$. Consider the arcs

$$
\gamma_{n, \lambda}=h_{\text {new }}^{n}\left(\beta_{0, \lambda} \times\{1\}\right), \quad n \in \mathbb{Z}, \quad \lambda \in \Lambda .
$$

The previous discussions imply that $\gamma_{n, \lambda}=\beta_{n, \lambda} \times\{1\}$ if $n \geq 0$. For negative $n$ the $\operatorname{arcs} \gamma_{n, \lambda}$ have the same end points as $\beta_{n, \lambda} \times\{1\}$ but the open $\operatorname{arcs} \dot{\gamma}_{n, \lambda}$ are contained in $\{\rho>1\}$. 


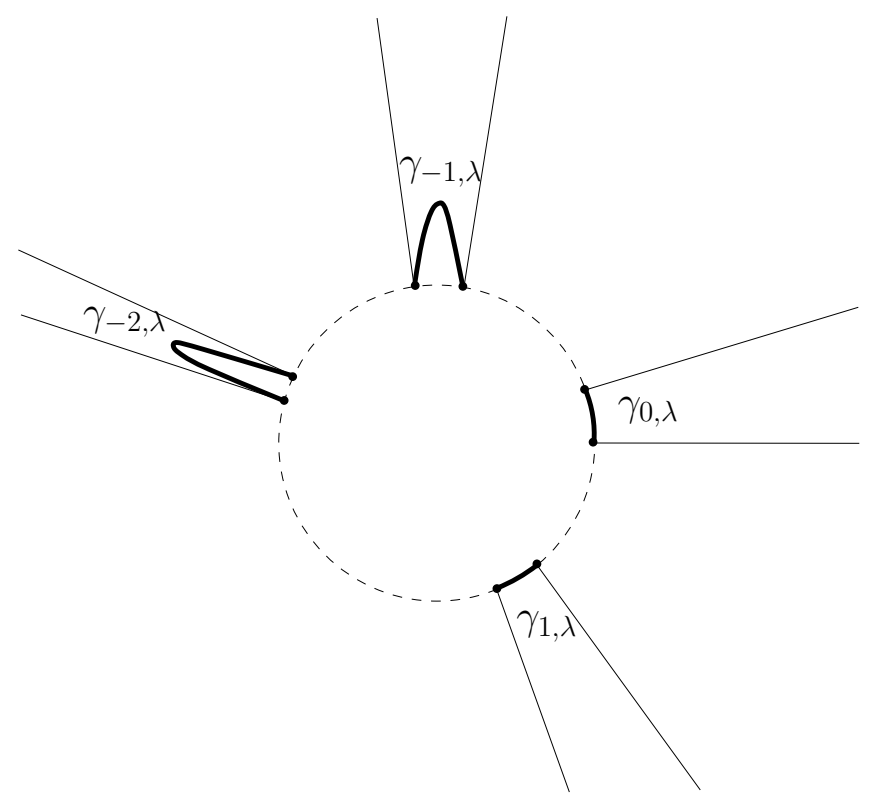

Going back to the definition of $h_{\text {new }}$ we observe that every orbit $\left\{\left(\bar{\theta}_{n}, \rho_{n}\right)\right\}$ with $\bar{\theta}_{0} \in \beta_{0, \lambda}$ and $\rho_{0}=1$ satisfies

$$
\rho_{n+1}=\frac{1}{2} \rho_{n}+\frac{1}{2}-\Omega_{\text {new }}\left(\bar{\theta}_{n}\right), n<0 .
$$

This recurrence leads to an explicit parametrization of the $\operatorname{arc} \gamma_{n, \lambda}$ as $\rho=$ $\sigma_{n, \lambda}(\bar{\theta})$ with

$$
\sigma_{n, \lambda}(\bar{\theta})=1+\sum_{h=0}^{|n|-1} 2^{h+1} \Omega_{n e w}\left(\phi^{h}(\bar{\theta})\right), \bar{\theta} \in \beta_{n, \lambda} .
$$

Finally we can define $\sigma$ as

$$
\sigma(\bar{\theta})= \begin{cases}\sigma_{n, \lambda}(\bar{\theta}) & \text { if } \bar{\theta} \in \beta_{n, \lambda}, n<0, \lambda \in \Lambda \\ 1 & \text { if } \bar{\theta} \in F_{+}\end{cases}
$$

To prove that $\sigma$ is unbounded it is sufficient to fix some $\lambda \in \Lambda$ and $\bar{\theta} \in \dot{\beta}_{0, \lambda}$ and notice that, for negative $n$,

$$
\sigma\left(\phi^{n}(\bar{\theta})\right) \geq 1+2 \sum_{h=0}^{|n|-1} \Omega\left(\phi^{-h}(\bar{\theta})\right) \rightarrow+\infty \text { as } n \rightarrow-\infty .
$$

To describe $\partial U_{\text {new }}$ we apply the following lemma dealing with domains whose boundary is a "Cantorian sun". 
Lemma 4. Let $C$ be a Cantor set in $\mathbb{T}$ and $\sigma: \mathbb{T} \rightarrow[1, \infty)$ a lower semicontinuous function that is continuous at each point in $\mathbb{T} \backslash C$ and satisfies

$$
\sigma\left(\bar{\theta}_{*}\right)=1, \quad \limsup _{\bar{\theta} \rightarrow \bar{\theta}_{*}} \sigma(\bar{\theta})=+\infty
$$

at each $\bar{\theta}_{*} \in C$. Then

$$
U=\{(\bar{\theta}, \rho)): \rho<\sigma(\bar{\theta})\}
$$

is an open and simply connected subset of $\mathbb{R}^{2}$ with connected boundary given by

$$
\partial U=\{(\bar{\theta}, \sigma(\bar{\theta}))): \bar{\theta} \in \mathbb{T}\} \cup\{(\bar{\theta}, \rho): \bar{\theta} \in C, \rho \geq 1\} .
$$

The proof of this lemma is straightforward and we do not give the details. However it remains to check that the function $\sigma$ defining $U_{\text {new }}$ satisfies the conditions of the lemma. The only delicate point is to prove that

$$
\limsup _{\bar{\theta} \rightarrow \bar{\theta}_{*}} \sigma(\bar{\theta})=+\infty \text { for each } \bar{\theta}_{*} \in C \text {. }
$$

This is more or less a repetition of a previous argument. We fix $\lambda \in \Lambda$ and $\bar{\theta} \in \dot{\beta}_{0, \lambda}$. Since the point $\bar{\theta}_{*}$ is in the $\alpha$-limit set of $\left\{\phi^{n}(\bar{\theta})\right\}$, there exists a sequence of integers $\tau(n) \rightarrow-\infty$ and such that $\phi^{\tau(n)}(\bar{\theta}) \rightarrow \bar{\theta}_{*}$. We know that $\sigma\left(\phi^{\tau(n)}(\bar{\theta})\right) \rightarrow+\infty$ and so the claim is proved.

Now we have a complete knowledge of the domain $U_{\text {new }}$ and it is easy to describe the space of prime ends. In this case there is a natural bijection between prime ends and accessible points of $\partial_{\mathbb{S}^{2}} U_{\text {new }}=\partial U_{\text {new }} \cup\{\infty\}$. Each prime end is defined via the radial arc

$$
\Gamma_{\bar{\theta}}: t \in[0,1] \mapsto(\bar{\theta}, t \sigma(\bar{\theta})) .
$$

Since $h_{\text {new }}$ transforms $\Gamma_{\bar{\theta}}$ into an arc equivalent to $\Gamma_{\phi(\bar{\theta})}$ we deduce that $h^{\star}$ is conjugate to $\phi$.

\section{Rotations do not appear on prime ends}

It is easy to construct homeomorphisms with an attractor such that the map induced on prime ends is a rotation. As an example consider the time 1 map $h=\phi_{1}$ associated to the flow $\left\{\phi_{t}\right\}_{t \in \mathbb{R}}$ defined by

$$
\dot{\rho}=\frac{\rho(\rho-1)(2-\rho)}{1+\rho^{2}}, \quad \dot{\theta}=\omega
$$

with $\omega \in \mathbb{R} \backslash \mathbb{Q}$. The map $h$ is dissipative since every orbits enters into the ball $\rho \leq 2+\epsilon$. Moreover the origin is asymptotically stable and the region of attraction is the disk $\rho<1$. It is easy to show that $h^{\star}$ is topologically conjugate to $R_{\omega}$. In this Section we prove that this cannot occur if $h$ is in the class $\mathcal{H}_{0}$. 
Theorem 5. Assume that $h \in \mathcal{H}_{0}$ and the rotation number $\rho(h, U)$ is irrational. Then the induced map $h^{\star}: \mathbb{P} \rightarrow \mathbb{P}$ is a Denjoy homeomorphism.

We need some preliminaries before the proof. First we state a result on General Topology taken from [12].

Lemma 6. Let $X$ be a connected topological space and let $A$ and $B$ be open and connected subsets of $X$ such that the boundaries $\partial A$ and $\partial B$ are connected and satisfy

$$
\partial A \neq \emptyset, \quad \partial B \neq \emptyset, \quad \partial A \cap \partial B=\emptyset .
$$

Then one of the following alternatives holds:

(i) $X=A \cup B$ (ii) $c l_{X} A \subset B$ (iii) $c l_{X} B \subset A$ (iv) $c l_{X} A \cap c l_{X} B=\emptyset$.

For the proof we refer to [12]. Next, we present some properties of prime ends.

Given $\mathfrak{p} \in \mathbb{P}$, a chain of crosscuts representing $\mathfrak{p}$ will be denoted by $\left(C_{n}\right)$. It is not restrictive to assume that $0 \notin C_{n}$. The component of $U \backslash C_{n}$ which does not contain the origin will be denoted by $V_{n}$.

Assume now that $\gamma$ is a crosscut in $U$ with end points $a \neq b$ lying on $\partial_{\mathbb{S}^{2}} U$. Let $V$ denote one of the components of $U \backslash \gamma$. We say that a prime end $\mathfrak{p}$ divides $V$ if there is a chain of crosscuts $\left(C_{n}\right)$ representing $\mathfrak{p}$ such that $V_{n}$ is contained in $V$ for each $n$. We employ the notation

$$
\tilde{V}=\{\mathfrak{p} \in \mathbb{P}: \mathfrak{p} \text { divides } V\} .
$$

It is known that $\tilde{V}$ is an open arc in $\mathbb{P}$. The closure of $\tilde{V}$ is a closed arc whose end points are the accessible prime ends determined by $\gamma$. Denote it by $\alpha_{V}=c l_{\mathbb{P}}(\tilde{V})$ and notice that $\alpha_{V}$ is a proper arc since its end points $\mathfrak{p}_{a}$ and $\mathfrak{p}_{b}$ are different.

Lemma 7. In the previous notations assume that $h \in \mathcal{H}_{0}$ and there exists a crosscut $\gamma$ in $U$ with

$$
h^{k}(V) \subset V
$$

for some $k \in \mathbb{Z} \backslash\{0\}$. Then $\rho(h, U)$ is rational.

Proof. Since $h$ is a homeomorphism, $\mathfrak{p}$ divides $V$ whenever $h^{\star}(\mathfrak{p})$ divides $h(V)$. In consequence $h^{\star}(\tilde{V})=\widetilde{h(V)}$ or, more generally,

$$
\left(h^{\star}\right)^{k}(\tilde{V})=\widetilde{h^{k}(V)} .
$$

The assumption $h^{k}(V) \subset V$ implies that $\widetilde{h^{k}(V)} \subset \tilde{V}$. Hence $\left(h^{\star}\right)^{k}(\tilde{V}) \subset \tilde{V}$ and, taking closures, $\left(h^{\star}\right)^{k}\left(\alpha_{V}\right) \subset \alpha_{V}$. Brouwer fixed point Theorem in 1 dimension implies that $h^{\star}$ has a periodic point and so $\rho$ must be a rational number of the type $\frac{n}{k}$. 
We are ready to prove the main result.

Proof of Theorem 5. The proof will consist in finding an open set $G$ in $\mathbb{P}$ with

$$
G \neq \emptyset, \quad\left(h^{\star}\right)^{k}(G) \cap G=\emptyset \text { if } k \in \mathbb{Z} \backslash\{0\} .
$$

This is not possible if all orbits are recurrent, as it is the case in an irrational rotation. To construct $G$ we proceed by steps.

Step 1. Construction of a crosscut $\gamma$ in $U$ with $\dot{\gamma} \cap h^{k}(\dot{\gamma})=\emptyset$ if $k \in$ $\mathbb{Z} \backslash\{0\}$.

In the following we will work with the extension of the map $h$ to the Riemann sphere with $h(\infty)=\infty$. The map being dissipative is equivalent to $\infty$ being a repeller, that is an asymptotically stable fixed point for $h^{-1}$. Let $W$ denote the region of repulsion of $\infty$,

$$
W=\left\{x \in \mathbb{S}^{2}: h^{n}(x) \rightarrow \infty \text { as } n \rightarrow-\infty\right\} .
$$

Then $W$ is a simply connected open subset of $\mathbb{S}^{2}$ and the restriction of $h$ to $W, h_{W}: W \rightarrow W$, is topologically conjugate to the dilation $\mathbb{C} \rightarrow \mathbb{C}, z \mapsto 2 z$ (see [9] and [5] for more details). Since $W$ is a neighborhood of $\infty$ and $U$ is unbounded, these two sets must have a non void intersection. Let $x_{0}$ be a point in $W \cap U$. Using $h_{W}$ is a topological dilation we can find an arc $\Gamma \subset W$ with end points at $x_{0}$ and $\infty$ and such that $\Gamma \subset h(\Gamma)$. It will be convenient to employ the natural ordering in $\Gamma$ having the minimum at $x_{0}$ and the maximum at $\infty$. In particular an open sub-arc of $\Gamma$ can be expressed as an interval $(a, b)_{\Gamma}$ with $a<b, a, b \in \Gamma$. Since $\Gamma$ is inside $W$ it is easy to describe the backward dynamics of $h$ on $\Gamma$. Given any $y_{0} \in \Gamma \backslash\{\infty\}$, the sequence $y_{n}=h^{n}\left(y_{0}\right)$ satisfies $y_{n} \in \Gamma$ if $n \leq 0$ and

$$
y_{0}<y_{-1}<y_{-2}<. .<y_{n}<. . \rightarrow \infty \text { as } n \rightarrow-\infty .
$$

We claim that $\Gamma$ is not contained in $U$, for otherwise we could apply Proposition 6 in [14] to deduce that the rotation number vanishes. Indeed the result in [14] dealt with positively invariant rays but the same proof works for negative invariance.

Let us fix some $y_{0} \in \Gamma \backslash U$. Since both orbits $y_{n}=h^{n}\left(y_{0}\right)$ and $x_{n}=h^{n}\left(x_{0}\right)$ converge to $\infty$ as $n \rightarrow-\infty$, there exist two negative integers $N$ and $M$ such that $y_{0}<x_{N}<y_{M}$. Let $J$ be the connected component of $\Gamma \cap U$ containing $x_{N}$.

Since $y_{0}$ and $y_{M}$ are not in $U$, the set $J$ must be an open sub-arc of $\Gamma$ with end points $a$ and $b, y_{0} \leq a<x_{N}<b \leq y_{M}$. Moreover, $a, b \in \partial U$. This arc has the important property

$$
J \cap h^{k}(J)=\emptyset \text { if } k<0 .
$$

To check this notice that $J=(a, b)_{\Gamma}$ and $h^{k}(J)=\left(h^{k}(a), h^{k}(b)\right)_{\Gamma}$. Since $a<h^{k}(a)$ and $b<h^{k}(b)$, a non-empty intersection would imply that $h^{k}(a)$ 


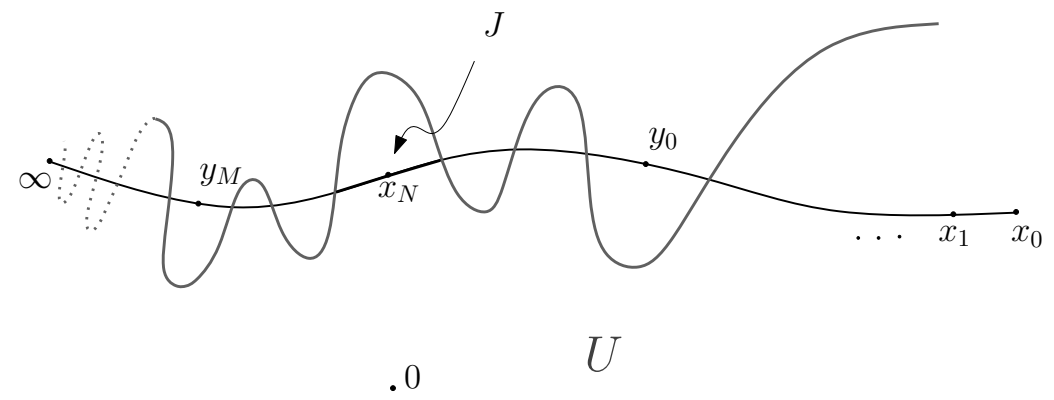

belongs to $J$. This is not possible since $J$ is contained in $U$. The goal of the first step is achieved taking $\gamma=J \cup\{a, b\}$. Notice that $J \cap h^{k}(J)=\emptyset$ is equivalent to $J \cap h^{-k}(J)=\emptyset$ and also that the origin cannot belong to $\gamma$. From now on $V$ is the connected component of $U \backslash \gamma$ with $0 \notin V$.

Step 2. $V \cap h^{k}(V)=\emptyset$ if $k \in \mathbb{Z} \backslash\{0\}$.

We apply Lemma 6 with $X=U, A=V$ and $B=h^{k}(V)$. Then $\partial A=\dot{\gamma}$ and $\partial B=h^{k}(\dot{\gamma})$. The condition $\partial A \cap \partial B=\emptyset$ follows from Step 1. Let us eliminate the first three alternatives. The alternative (i) cannot hold because the origin is not int $V \cup h^{k}(V)$. Assume now that (ii) or (iii) holds, then either $h^{-k}(V) \subset V$ or $h^{k}(V) \subset V$. Then we could apply Lemma 7 to conclude that $\rho$ is rational. In conclusion (iv) must hold and so the claim of Step 2 is valid.

Step 3. $\tilde{V} \cap\left(h^{\star}\right)^{k}(\tilde{V})=\emptyset$ if $k \in \mathbb{Z} \backslash\{0\}$.

Let $\mathfrak{p}$ be a prime end in $\tilde{V}$. Then every sequence of crosscuts defining $\mathfrak{p}$ must eventually enter into $V$. This means that $V_{n} \subset V$ for large $n$. If $\mathfrak{p}$ would also belong to $\left(h^{\star}\right)^{k}(\tilde{V})=\widetilde{h^{k}(V)}$ then the sequence of crosscuts should also enter into $h^{k}(V)$, meaning that $V_{n} \subset h^{k}(V)$ for large $n$. Since we know that $V \cap h^{k}(V)=\emptyset$ such a prime end cannot exist.

The proof is complete since we can take $G=\tilde{V}$.

Remark 8. The previous proof leads to a result slightly more general than Theorem 5.

"Let $h: \mathbb{R}^{2} \rightarrow \mathbb{R}^{2}$ be an orientation-preserving, dissipative homeomorphism with $h(0)=0$. Let $U$ be a proper, unbounded and simply connected open subset of $\mathbb{R}^{2}$ with $0 \in U$ and $h(U)=U$. Assume that $\rho(h, U)$ is irrational, then $h^{\star}: \mathbb{P} \rightarrow \mathbb{P}$ is a Denjoy homeomorphism."

Notice that 0 is not necessarily an attractor and $U$ can contain more fixed points.

\section{Periodic points and irrational rotation numbers}

Given a homeomorphism $h$ of the plane the set of periodic points is

$$
\operatorname{Per}(h)=\left\{x \in \mathbb{R}^{2}: h^{n}(x)=x \text { for some } n \neq 0\right\} .
$$


It was proved in [14] that every $h$ in $\mathcal{H}_{0}$ with rational rotation number has periodic points in $\mathbb{R}^{2} \backslash U$. The examples constructed in Sections 3 and 4 with irrational rotation number satisfy $\operatorname{Per}(h)=\{0\}$. As mentioned in the introduction we conjecture that this is a characterization of $\rho(h, U) \in$ $\mathbb{R} \backslash \mathbb{Q}$ in the class $\mathcal{H}_{0}$. By now we can prove the result under an additional assumption: the point of infinity is accessible from the region of attraction. Notice that this condition holds for the examples in Section 3 but not for those in Section 4.

Theorem 9. Let $h$ be a map in $\mathcal{H}_{0}$ such that $\infty$ is accessible from $U$. Then the following statements are equivalent:

(i) $\rho(h, U)$ is irrational.

(ii) $\operatorname{Per}(h)=\{0\}$.

For the proof we need a preliminary result on dynamics in $\mathbb{T}$.

Lemma 10. Assume that $\phi: \mathbb{T} \rightarrow \mathbb{T}$ is an orientation-preserving homeomorphism with irrational rotation number and let $B$ be a compact subset of $\mathrm{T}$ with $B \subset \phi(B)$. In addition assume that there exists some $\bar{\theta} \in \mathrm{T}$ such that $B$ is contained in the closed arc with end points $\bar{\theta}$ and $\phi(\bar{\theta})$. Then $B$ is the empty set.

We prove this lemma in the Appendix.

Proof of Theorem 9. (ii) $\Rightarrow$ (i) This is a consequence of Proposition 2 in [14]. If (i) does not hold then $\rho=\frac{m}{n} \in \mathbb{Q}$ and so the map $h^{n}$ satisfies $h^{n}(U)=U$ and $\rho\left(h^{n}, U\right)=0$. Then $h^{n}$ has a fixed point in $\mathbb{R}^{2} \backslash U$ and so (ii) does not hold. Notice that we do not need to assume that $\infty$ is accessible from $U$ in the previous argument.

(i) $\Rightarrow$ (ii) By a contradiction argument assume that $y_{0}$ is a fixed point in $\mathbb{R}^{2} \backslash U$. In principle the contradiction argument would lead to the existence of a periodic point in $\mathbb{R}^{2} \backslash U$ but then we can replace $h$ by $h^{N}$ for suitable $N$. We structure the proof by steps.

Step 1. There exist two arcs $\gamma_{0}$ and $\gamma_{1}$ going from 0 to $\infty$ and satisfying

- $\gamma_{i} \backslash\{\infty\} \subset U, i=0,1$.

- $\gamma_{0} \cap \gamma_{1}=\{0, \infty\}$.

- Let $\infty_{0}$ and $\infty_{1}$ be the prime ends determined by $\gamma_{0}$ and $\gamma_{1}$, then $h^{\star}\left(\infty_{0}\right)=\infty_{1}$.

By assumption $\infty$ is accessible from $U$ and so there is an arc $\gamma$ going from 0 to $\infty$ such that $\gamma \backslash\{\infty\} \subset U$. This arc determines a prime end, say $\infty_{0} \in \mathbb{P}$. From (i) we know that $\rho$ is irrational so $h^{\star}$ has not fixed point. In particular $h^{\star}\left(\infty_{0}\right) \neq \infty_{0}$ and $h^{\star}\left(\infty_{0}\right)$ is the prime end determined by the 

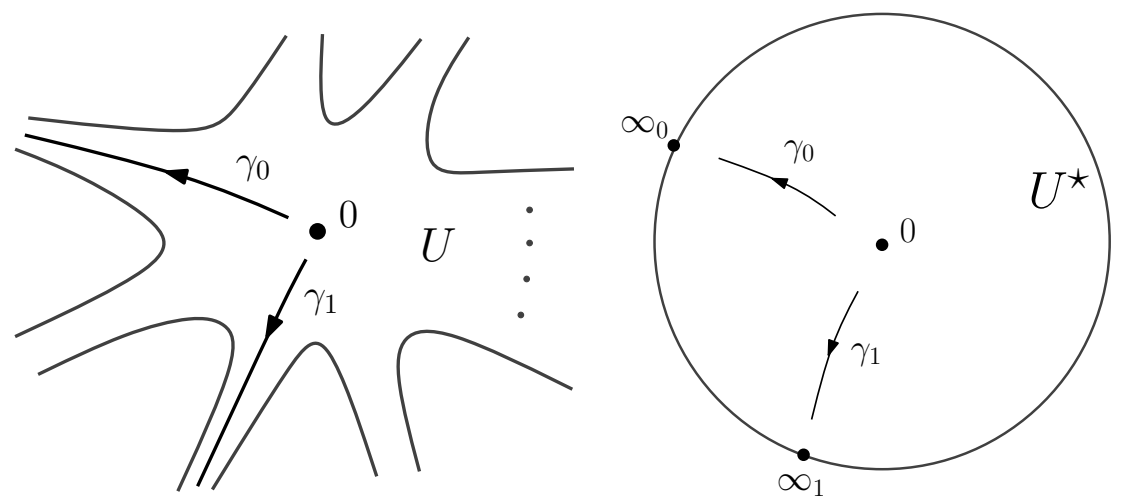

Figure 3: Arcs constructed in Step 1.

arc $h(\gamma)$. In general the arcs $\gamma$ and $h(\gamma)$ cannot be chosen as $\gamma_{0}$ and $\gamma_{1}$ since it could occur that they have some crossings, as it is indicated in the next figure.
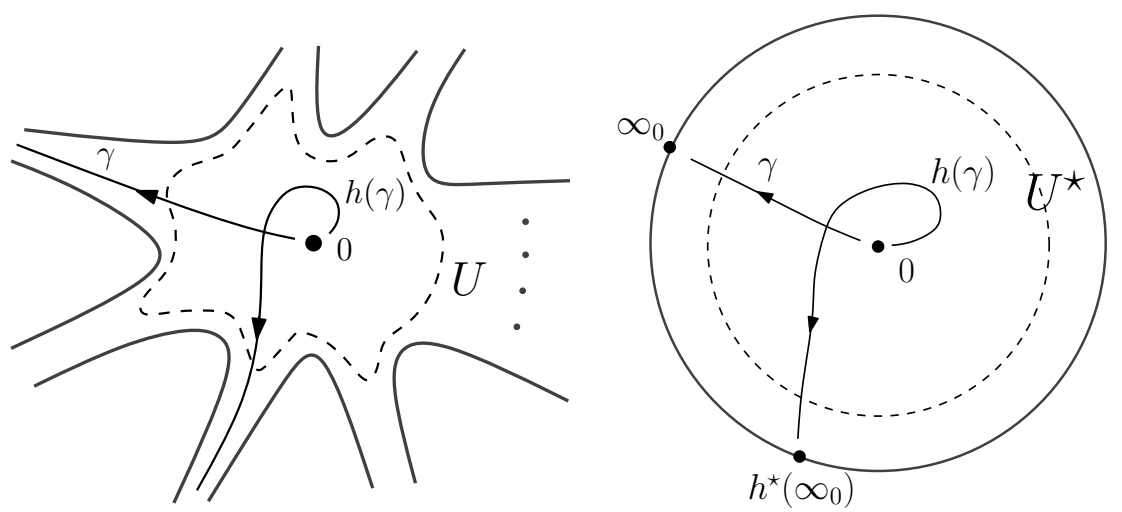

The arcs in $U^{\star}$ defined as $(\gamma \backslash\{\infty\}) \cup\left\{\infty_{0}\right\}$ and $(h(\gamma) \backslash\{\infty\}) \cup\left\{h^{\star}\left(\infty_{0}\right)\right\}$ connect the origin to $\infty_{0}$ and $\infty_{1}:=h^{\star}\left(\infty_{0}\right)$ respectively. Since these two points of $\mathbb{P}$ do not coincide, it is possible to find a disk centered at the origin of $U^{\star}$ such that the two arcs do not meet outside the disk. Going back to $U$ via the Riemann map we find a topological disk $\Delta \subset U$ with $0 \in \operatorname{int}(\Delta)$ and $[\gamma \cap h(\gamma)] \backslash\{\infty\} \subset \operatorname{int}(\Delta)$.

The arcs $\gamma$ and $h(\gamma)$ can be modified inside $\Delta$ in order to get new arcs $\gamma_{0}$ and $\gamma_{1}$ from 0 to $\infty$, contained in $U \backslash\{\infty\}$ and such that

- $\gamma_{0} \cap\left(\mathbb{S}^{2} \backslash \Delta\right)=\gamma \cap\left(\mathbb{S}^{2} \backslash \Delta\right)$

- $\gamma_{1} \cap\left(\mathbb{S}^{2} \backslash \Delta\right)=h(\gamma) \cap\left(\mathbb{S}^{2} \backslash \Delta\right)$

- $\gamma_{0} \cap \gamma_{1}=\{0, \infty\}$. 
By construction these arcs determine the same prime ends as $\gamma$ and $h(\gamma)$.

Step 2. The set $A=\left(\gamma_{0} \cup \gamma_{1}\right) \backslash\{\infty\}$ decomposes $\mathbb{R}^{2}$, as well as $U$, in two connected components

$$
\mathbb{R}^{2} \backslash A=\Lambda_{1} \cup \Lambda_{2}, \quad U \backslash A=\Omega_{1} \cup \Omega_{2} .
$$

Moreover, $\Omega_{i}=\Lambda_{i} \cap U, i=1,2$.

The Jordan curve $J=\gamma_{0} \cup \gamma_{1}$ splits $\mathbb{S}^{2}$ in two components $\Lambda_{1}$ and $\Lambda_{2}$. Thus,

$$
\mathbb{R}^{2} \backslash A=\mathbb{S}^{2} \backslash J=\Lambda_{1} \cup \Lambda_{2}
$$

Looking at the arc $A \cup\left\{\infty_{0}, \infty_{1}\right\}$ of $U^{\star}$ we observe that also $U$ is split by $A$ in two components $\Omega_{1}$ and $\Omega_{2}$. The Jordan-Schönflies Theorem applied to $J$ implies that $\Lambda_{i} \cap U \neq \emptyset$ for $i=1,2$. The sets $\Omega_{i}$ are connected and contained in $\mathbb{R}^{2} \backslash A$. Perhaps after labelling the sets again we get the inclusions $\Omega_{i} \subset \Lambda_{i}, i=1,2$. An easy argument now shows that $\Omega_{i}$ is indeed the intersection of $\Lambda_{i}$ and $U$.

Step 3. There exists a continuum $K$ connecting $y_{0}$ and $U$ and satisfying

$$
h^{-1}(K) \subset K, \quad K \cap\left[\gamma_{0} \cup \gamma_{1}\right]=\emptyset .
$$

The restriction of $h$ to the region of attraction $U$ is topologically conjugate to the contraction $\mathbb{C} \rightarrow \mathbb{C}, z \mapsto \frac{1}{2} z$. Hence we can find a topological disk $D_{0}$ with

$$
D_{0} \subset U, \quad 0 \in \operatorname{int}\left(D_{0}\right), \quad h\left(D_{0}\right) \subset \operatorname{int}\left(D_{0}\right) .
$$

Similarly we know that the restriction of $h$ to the region of repulsion $W$ of $\infty$ is conjugate to the dilation $z \mapsto 2 z$. This allows us to find a second topological disk $D_{\infty}$ disjoint with $D_{0}$ and satisfying

$$
D_{\infty} \subset W, \infty \in \operatorname{int}_{\mathbb{S}^{2}}\left(D_{\infty}\right), \quad D_{\infty} \subset h\left(\operatorname{int}_{\mathbb{S}^{2}}\left(D_{\infty}\right)\right) .
$$

The points in $D_{0}$ are attracted by the origin as $n \rightarrow+\infty$ while the points in $D_{\infty}$ come from $\infty$. This implies that the fixed point $y_{0}$ cannot be in any of the two disks. The set

$$
\tilde{K}=\bigcap_{j \geq 0} h^{j}\left(\mathbb{R}^{2} \backslash i n t_{\mathbb{S}^{2}}\left(D_{\infty}\right)\right)
$$

is an invariant continuum connecting 0 and $y_{0}$.

Let $K_{0}$ be the component of $\tilde{K} \backslash \operatorname{int}\left(D_{0}\right)$ containing $y_{0}$. This set is negatively invariant and connects $y_{0}$ and $U$ but it could meet the $\operatorname{arc} \gamma_{0} \cup \gamma_{1}$. To exclude this possibility we consider a negative iterate $K=h^{-N}\left(K_{0}\right)$ with $N$ large enough. We claim that this continuum satisfies all the required properties. $K_{0}$ is a continuum connecting $y_{0}$ to $\partial D_{0}$ and so $K$ connects $y_{0}$ 
to $h^{-N}\left(\partial D_{0}\right)$, a subset of $U$. The property of negative invariance is inherited from $K_{0}$. To prove that $K$ does not intersect $\gamma_{0} \cup \gamma_{1}$ we observe that the compact set $\tilde{K} \cap\left(\gamma_{0} \cup \gamma_{1}\right)$ is contained in $U$ and so it is uniformly attracted by the origin. We select $N$ so that $h^{N}\left(\tilde{K} \cap\left(\gamma_{0} \cup \gamma_{1}\right)\right)$ lies inside $\operatorname{int}\left(D_{0}\right)$. This excludes the possibility of having a point $x$ in $K \cap\left(\gamma_{0} \cup \gamma_{1}\right)$, for otherwise $y=h^{N}(x)$ should lie simultaneously in $K_{0}$ and $\operatorname{int}\left(D_{0}\right)$.

Step 4. The set

$$
B=c l_{U^{\star}}(K \cap U) \cap \mathbb{P}
$$

is non-empty, compact and negatively invariant under $h^{\star}$.

From the previous step we know that $K \cap U \neq \emptyset$ and also $K \cap \partial U \neq \emptyset$. Since $K$ is a continuum we can find a sequence $z_{n} \in K \cap U$ accumulating on $\partial U$. The accumulation points of $\left\{z_{n}\right\}$ in $U^{\star}$ must belong to $\mathbb{P}$. This shows that $B$ is non-empty. This set is obviously compact and due to the negative invariance of $K$ under $h$,

$$
h^{\star}(B)=c l_{U^{\star}}(h(K) \cap U) \cap \mathbb{P} \supset B .
$$

Conclusion. We are ready to arrive at a contradiction. The continuum $K$ must be included in one of the components of $\mathbb{R}^{2} \backslash A$, say $\Lambda_{1}$. Then $K \cap U \subset \Lambda_{1} \cap U=\Omega_{1}$ and all the prime ends in $B$ are inside the arc $\alpha_{\Omega_{1}}=$ $c l_{\mathbb{P}}\left(\tilde{\Omega}_{1}\right)$ determined by the crosscut $A$. This arc has $\infty_{0}$ and $\infty_{1}=h^{\star}\left(\infty_{0}\right)$ as end points and, according to Lemma 10, the set $B$ should be empty. This is against Step 4 .

\section{A Appendix}

Proof of Lemma 3. It is enough to prove the result for some map $\hat{\phi}$ conjugate to $\phi$. Following Section 2 we consider the double sequence of arcs $\left(\beta_{n, \lambda}\right)_{n \in \mathbb{Z}, \lambda \in \Lambda}$ such that $\mathbb{T} \backslash C$ is the disjoint union of the open $\operatorname{arcs} \dot{\beta}_{n, \lambda}$ and $\phi\left(\beta_{n, \lambda}\right)=\beta_{n+1, \lambda}$. For convenience we assume that $\Lambda$ is a subset of $\mathbb{N} \backslash\{0\}$. After lifting the $\operatorname{arcs} \beta_{n, \lambda}$ and $\beta_{n+1, \lambda}$ to compact intervals of $\mathbb{R}$, we consider the unique homeomorphism $A_{n, \lambda}: \beta_{n, \lambda} \rightarrow \beta_{n+1, \lambda}$ whose lift is an increasing affine map. In particular,

$$
\frac{\operatorname{dist}_{\mathbb{T}}\left(A_{n, \lambda}(\bar{\theta}), C\right)}{\text { length }\left(\beta_{n+1, \lambda}\right)}=\frac{\operatorname{dist}(\bar{\theta}, C)}{\text { length }\left(\beta_{n, \lambda}\right)} \quad \text { if } \bar{\theta} \in \beta_{n, \lambda} .
$$

Next we define the modification of $\phi$,

$$
\hat{\phi}(\bar{\theta})= \begin{cases}\phi(\bar{\theta}) & \text { if } \bar{\theta} \in C \\ A_{n, \lambda}(\bar{\theta}) & \text { if } \bar{\theta} \in \beta_{n, \lambda} .\end{cases}
$$

It can be proved that $\hat{\phi}: \mathbb{T} \rightarrow \mathbb{T}$ is an orientation-preserving homeomorphism. Since $\phi$ and $\hat{\phi}$ coincide in $C$ the new map is also a Denjoy map with 
the same rotation number. Possibly the two maps do not coincide in the interior of the $\operatorname{arcs} \beta_{n, \lambda}$ but

$$
\phi\left(\beta_{n, \lambda}\right)=\hat{\phi}\left(\beta_{n, \lambda}\right) .
$$

This implies that if $\mathcal{P}: \mathbb{T} \rightarrow \mathbb{T}$ is a Cantor function associated to $C$ with $\mathcal{P} \circ \phi=R_{\omega} \circ \mathcal{P}$, then $\mathcal{P} \circ \hat{\phi}=R_{\omega} \circ \mathcal{P}$ is also valid. In consequence Markley's invariant $\mathcal{P}(A)$ is common to $\phi$ and $\hat{\phi}$ and these maps are conjugate. Define

$$
\Omega(\bar{\theta})= \begin{cases}0 & \text { if } \bar{\theta} \in C \\ \frac{1}{\lambda(|n|+1)} \frac{\operatorname{dist}_{\mathbb{T}}(\bar{\theta}, C)}{\text { length }\left(\beta_{n, \lambda}\right)} & \text { if } \bar{\theta} \in \beta_{n, \lambda} .\end{cases}
$$

We are going to check that this function satisfies the conditions of the lemma when $\phi$ is replaced by $\hat{\phi}$. The conditions (i) and (ii) are obvious. Notice that $0 \leq \Omega \leq \frac{1}{2}$ everywhere. To check (iii) we pick any point $\bar{\theta}_{0}$ in some arc $\beta_{N, \lambda}$. Using the property (3) several times we arrive at

$$
\frac{\operatorname{dist}_{\mathbb{T}}\left(\hat{\phi}^{n}\left(\bar{\theta}_{0}\right), C\right)}{\text { length }\left(\beta_{N+n, \lambda}\right)}=\frac{\operatorname{dist}_{\mathbb{T}}\left(\bar{\theta}_{0}, C\right)}{\text { length }\left(\beta_{N, \lambda}\right)}, n \in \mathbb{Z} .
$$

In consequence

$$
\Omega\left(\hat{\phi}^{n}\left(\bar{\theta}_{0}\right)\right)=\frac{|N|+1}{|N+n|+1} \Omega\left(\bar{\theta}_{0}\right) .
$$

This proves (iii) since $\sum \Omega\left(\hat{\phi}^{n}\left(\bar{\theta}_{0}\right)\right)$ leads to the harmonic series whenever $\bar{\theta}_{0} \in \dot{\beta}_{N, \lambda}$.

To complete the proof we must check the continuity of $\Omega$ at each point of $\mathbb{T}$. This is obvious for points lying in some open arc $\dot{\beta}_{n, \lambda}$. Let us take a point in $\bar{\theta}$ in $C$ and a sequence $\left\{\bar{\theta}_{r}\right\}$ converging to $\bar{\theta}$, we must prove that $\Omega\left(\bar{\theta}_{r}\right) \rightarrow 0$. Since $\Omega$ vanishes on $C$ it is sufficient to consider sequences in the complement $\mathbb{T} \backslash C$, say $\bar{\theta}_{r} \in \dot{\beta}_{n(r), \lambda(r)}$ for each $r$. If $\bar{\theta}$ is in the inaccessible set $I$ then the length of the $\operatorname{arcs} \beta_{n(r), \lambda(r)}$ has to go to zero and so

$$
\max \{|n(r)|, \lambda(r)\} \rightarrow+\infty \text { as } r \rightarrow+\infty .
$$

In consequence

$$
\Omega\left(\bar{\theta}_{r}\right) \leq \frac{1}{2 \lambda(r)(|n(r)|+1)} \rightarrow 0 .
$$

This proves the continuity at points in $I$. When $\bar{\theta}$ is in $A$, the accessible set, it is convenient to discuss the continuity from each side of the point. The point $\bar{\theta}$ being an end point of some $\beta_{n, \lambda}$, the continuity from the side of this arc is automatic. From the other side the continuity is discussed as in the inaccessible case. 
Proof of Lemma 10. By a contradiction argument assume that $B$ contains at least one point, say $\bar{\theta} \in B$. Then $B$ also contains the backward orbit $\left\{\phi^{n}(\bar{\theta})\right\}_{n \leq 0}$ and the $\alpha$-limit set $L_{\alpha}(\bar{\theta})$. Here we have used that $B$ is negatively invariant and compact. If $L_{\alpha}(\bar{\theta})=\mathbb{T}$ we have reached already a contradiction because $B$ is contained in an arc. From now on we assume that $L_{\alpha}(\bar{\theta})$ is a Cantor set. Let $\gamma$ be the arc with end points $\bar{\theta}$ and $\phi(\bar{\theta})$ such that $\dot{\gamma} \cap B=\emptyset$. Then $\phi(\dot{\gamma}) \cap \phi(B)=\emptyset$ implying that $\phi(\dot{\gamma}) \cap B=\emptyset$. In general,

$$
\dot{\gamma}_{n} \cap B=\emptyset, \quad n \geq 0,
$$

where $\gamma_{n}=\phi^{n}(\gamma)$. The arcs $\gamma_{n}$ and $\gamma_{n+1}$ have a common end point, namely $\phi^{n+1}(\bar{\theta})$, and so $\Gamma=\bigcup_{n \geq 0} \gamma_{n}$ is a connected subset of $\mathbb{T}$. We claim that $\Gamma \neq \mathbb{T}$. Indeed $\Gamma \cap B$ is at most countable while $B$ is uncountable since it contains a Cantor set. At this point we can say that $\Gamma$ is homeomorphic to an interval of $\mathbb{R}$ (closed, open or half-open) and $\phi(\Gamma) \subset \Gamma$. Taking some point $\bar{\varphi} \in \Gamma$ we notice that $\left\{\phi^{n}(\bar{\varphi})\right\}_{n \geq 0}$ must converge to some point in the closure of $\Gamma$. This point should be fixed and this is the searched contradiction since we are assuming that the rotation number is irrational.

\section{References}

[1] D.K. Arrowsmith, C.M. Place, An Introduction to Dynamical Systems, Cambridge University Press, Cambridge, 1992

[2] K.T. Alligood, J.A. Yorke, Accessible saddles on fractal basin boundaries, Ergod. Th. Dynam. Sys. 12 (1992) 377-400.

[3] M. Barge, Prime end rotation numbers associated with the Hénon map. Continuum theory and dynamical systems, 15-33, Lecture Notes in Pure and Appl. Math., 149, Dekker, New York, 1993.

[4] G.D. Birkhoff, Sur quelques courbes fermées remarquables, Bull. Soc. Math. de France 60 (1932) 1-26.

[5] C. Bonatti, B. Kolev, Surface homeomorphisms with zero-dimensional singular set, Topology Appl. 90 (1998) 69-95.

[6] M. Bonino, A Brouwer-like theorem for orientation reversing homeomorphisms of the sphere, Fund. Math. 182 (2004) no. 1, 1-40.

[7] M.L. Cartwright, J.E. Littlewood, Some fixed point theorems, Annals of Math. 54 (1951) 1-37.

[8] J.K. Hale, Asymptotic behavior of dissipative systems. Mathematical Surveys and Monographs, 25. American Mathematical Society, Providence, RI, 1988. 
[9] B. Kerékjártó, Sur le caractèr topologique des représentations conformes, C.R. Acad. Sci. 198 (1934) 317-320.

[10] N. Levinson, Transformation theory of non-linear differential equations of the second order, Ann. of Math. 45 (1944) 723-737. Addendum: Ann. of Math. 49 (1948) 738.

[11] N.G. Markley, Homeomorphisms of the circle without periodic points, Proc. London Math. Soc. 20 (1970) 688-698.

[12] J.N. Mather, Topological proofs of some purely topological consequences of Carathéodory's theory of prime ends. Selected Studies. North Holland Publis. Co Eds. Th.M. Rassias, G.M. Rassias (1982), 225-255.

[13] S. Matsumoto, H. Nakayama, Continua as minimal sets of homeomorphisms of $\mathbb{S}^{2}$. arXiv:1005.0360v1 [math.DS].

[14] R. Ortega, F.R. Ruiz del Portal, Attractors with vanishing rotation number, J. Eur. Math. Soc. (to appear), www.ugr.es/ ecuadif/fuentenueva.htm.

[15] Ch. Pommerenke, Boundary behaviour of conformal maps, Lecture Notes in Math., Springer-Verlag 1991. 